\title{
Design and Implementation of Agricultural Information Service Platform Based On Streaming Media Technology
}

\author{
Xinlei Jin ${ }^{1, a^{*}}$, Na Zhang ${ }^{1, b}$ \\ ${ }^{1}$ Department of CCIE, Beijing University of Agriculture, North Agricultural Road No.7, Changping, \\ Beijing \\ ajinxinlei0000@163.com,zhangna@bua.edu.cn \\ *Xinlei Jin
}

Keywords: Agricultural informatization, Streaming media, Internet technology

\begin{abstract}
With the development of science and technology, for the corresponding "Internet plus" agriculture, this paper puts forward based on streaming media technology "agricultural information service platform" design, introduces the design and implementation of agricultural information service platform of the streaming media technology based on a platform, respectively from the three parts to elaborate design and overall design and database design. Includes the backstage management system using the platform service users and administrators of the platform, including user management, policy management, information management and other functions, greatly facilitate the service learning and the training of agricultural knowledge, learning efficiency.
\end{abstract}

\section{Introduction}

With the continuous development of Internet technology, increasing the bandwidth of the multimedia spread on the network is becoming more and more popular, especially for agricultural information of audio / video training more and more get the attention of people using the internet. In order to avoid the long wait time of media files, streaming media, such as streaming media, came into being. The use of efficient compression code technology, the multimedia file to effectively control the quality and resource share, using the cache system, with limited bandwidth, the user can watch the side edge of the cache, is a high-tech product of the progress of the times, to promote the development of the internet. The use of streaming media technology to build agricultural information service platform, will effectively promote the popularization of knowledge science, improve communication efficiency, accelerate agricultural information.

\section{Platform Function Design}

This platform is based on streaming media technology, the use of ASP.NET as the development language, for the vast number of users in the field of agricultural development of agricultural knowledge service platform. As a result of the streaming media, it can be used for the rapid transmission of large media files. Coupled with the main users of the platform for agricultural workers, for the user's characteristics, the platform requires simple interface, smooth operation.

Agricultural information dissemination platform user rights are divided into two main categories, one for the service platform administrator, the other is the platform for the service of ordinary users. 
The administrator can maintain a series of functions of the platform, such as the maintenance of the real-time policy information, the maintenance of the agricultural science knowledge, the maintenance of the message of the platform and so on. For ordinary users, the service platform, can effectively and quickly learned that the current policy, and query, learning in the field of agricultural planting and breeding technology, and use the platform provided by the message function, technical discussion.

\subsection{Policy Announcement Module}

The policy announcement function module is public function module, an open, without the user login, you can browse in the module, the new national policies and local policies, and to provide a fuzzy search function for the user, allowing users in the process of retrieving information, more convenient and efficient.

\subsection{Science and Technology Training Function Module}

Users in the use of science and technology training function module, the need for authentication, that is, registered login. This design can play a certain role in the protection of intellectual property rights of platform training materials. After verification by the user, will enter the main interface of science and technology training, training materials are divided into three categories, namely, text picture class, video class, practical technology"."The text picture" as described above, the training data in the form of graphic, intuitive description; "video" is based on the form of video media, spread of agricultural technology, such as planting / breeding technology methods; "practical technology" is the management of agriculture will follow experts in the field, shooting some field guide farmers pests identification and prevention of video media, and upload and sharing platform, for everyone to learn from the training modules together.

\subsection{Message Function Module}

Users in the use of the message function, the same is the need for login authentication, easy for each other to confirm the authenticity of the message and the maintenance of the platform. The user can pass the message to some of their interest in the problems of agriculture, other users can carry on the reply answer at the same time, the platform management will make some suggestions for the reply and reference.

\subsection{Administrator Maintenance Function}

Administrators use the platform to manage the background, the platform users, policy information, training information, message information, such as a series of management and maintenance.

\section{Platform Overall Structure Design}

\subsection{Platform Framework}

The service platform can be divided into two parts: foreground and background. The front desk is mainly for the general user to provide a series of agricultural services, such as access to policy announcements, learning agricultural knowledge, message exchange, etc., the background is the administrator of a variety of platform information maintenance and management. The overall framework of the platform is shown in figure 1. 


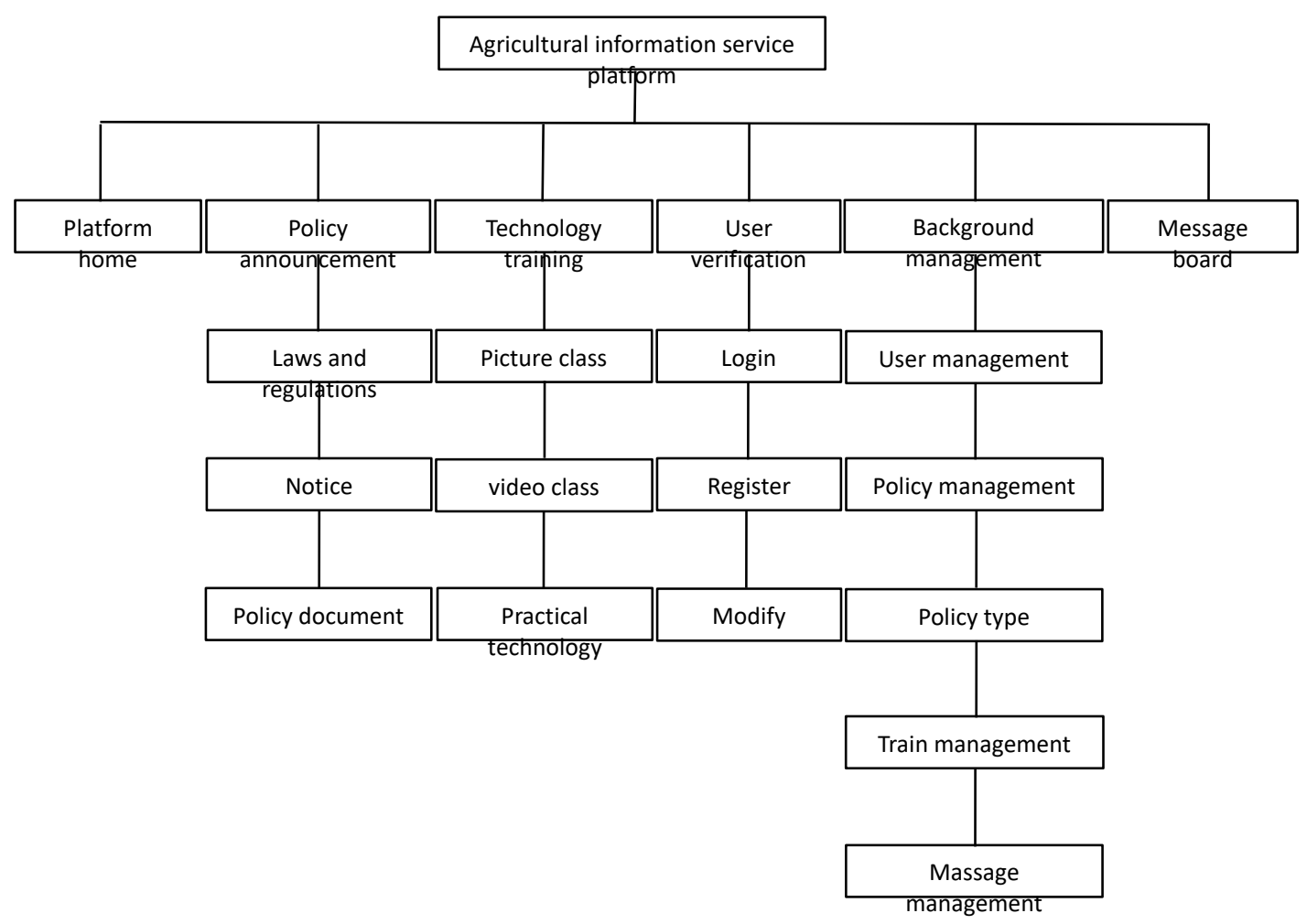

Figure 1 the overall framework of the platform

\subsection{Key Technologies}

\subsubsection{Hardware and software environment}

(1)Hardware environment and configuration: computer hardware of the client the system does not have specific requirements, the configuration of computer network, the higher the better the performance, the customer is in operation, the performance of the system is to show ${ }^{[1]}$.

(2)Microsoft Visual Studio (referred to as VS) is a basic set of complete development tools, written in the target code applies to all platforms supported by Microsoft. Visual Basic, Visual C++, Visual C\# and Visual J\# all use the same integrated development environment (IDE), the use of this IDE can share tools and contribute to the creation of mixed language solutions. In addition, these languages take advantage of the functionality of.NET Framework, through this framework can be used to simplify ASP Web applications and XML Web Services development of key technologies ${ }^{[2]}$.

(3)SQL Server is a relational database management system (DBMS), which was released and popularized by Microsoft in 2012, which provides a more comprehensive database platform. Its advantages include: strong function, high performance, good security, easy to operate, easy to maintain, and so on, it has a strong ability in enterprise support, business intelligence applications, management and development efficiency. It is based on the client / server model of a new generation of large relational database management system, is one of the most widely used $\mathrm{DBMS}^{[3]}$.

(4)ASP.Net programming is based on the common language of the framework developed by Microsoft Corporation, ASP.Net is almost entirely based on components and modular, can be developed by Microsoft visual studio.Net, Dreamwaver and other tools, greatly accelerate the development efficiency and efficiency, has good scalability ${ }^{[4]}$. 


\subsubsection{Streaming media selection}

Because of the rapid development of streaming media and the wide range of the media, the form of streaming media is diversified. The four main features of streaming media are the file volume, bit rate, coding standard, and the player. Streaming media is widely popular application of RMVB, ASF, WMV, MOV, FLV and so on, and each kind of media stream compression encoding standard used is different, in which MPEG-4, H.263 and H.264 three encoding standard is now widely recognized streaming media industry.

Because MPEG-4 only deal with the difference between the frame and the frame of the image, and the same elements are discarded, the volume of the synthesized multimedia file is greatly reduced. At the same bit rate, MPEG-4 can guarantee a higher quality of audio and video, which makes it possible to transmit high quality video and audio on a low bandwidth channel.

H.263 and H.264 video encoding standards are lower than 64KB / s transmission in the narrow bandwidth of communication channel, both using hybrid coding, and support the original image resolution, image shape and the clock frequency can also make many choices, broaden the scope of application. Expansion of the H.263 and H.264 is also powerful, it allows multi rate, multi rate and multi resolution, enhanced video information in easy error, easily lost packet transmission in heterogeneous network environment, ensuring the stability of the video. In addition, H.263 and H.264 image segmentation can also be limited to reduce error propagation. With the rapid development of mobile Internet in recent years, H.263 also did not let their own backward, and the mobile terminal perfect docking, mobile devices are supported by H.263 decoding, making it more compatible.

Through the comparison of several forms of streaming media, and the actual needs of the platform, it is found that the FLV streaming media can be effectively combined with the characteristics of the platform. The use of video media files, such as the length of the format of the conversion contrast, as shown in table 1.

Table 1 Comparison of different formats of streaming media file

\begin{tabular}{|c|c|c|c|c|c|}
\hline $\begin{array}{c}\text { Format } \\
\text { Features }\end{array}$ & RMVB & ASF & MOV & WMV & FLV \\
\hline File volume & $22.3 \mathrm{MB}$ & $28.6 \mathrm{MB}$ & $27.9 \mathrm{MB}$ & $35.9 \mathrm{MB}$ & $25.4 \mathrm{MB}$ \\
\hline Bit rate & 303 & 388 & 380 & 494 & 355 \\
\hline $\begin{array}{c}\text { Coding } \\
\text { standard }\end{array}$ & MPEG-4 & MPEG-4 & H.264 & WMV & H.263 \\
\hline Player & RealMedia & $\begin{array}{c}\text { Windows Media } \\
\text { Video }\end{array}$ & Quicktime & $\begin{array}{c}\text { Windows Media } \\
\text { Video }\end{array}$ & - \\
\hline
\end{tabular}

Flash Video referred to as FLV, along with the continuous development and innovation of Flash MX, FLV streaming media file format arises at the historic moment. The appearance of FLV makes it easier and easier to watch the audio and video media files on the network. The main characteristic is that the loading speed is very fast and the file space is very small. Under normal circumstances, the audio and video media files into the Flash, it will export a large file size of the SWF file, but the emergence of FLV is a good solution to this shortcoming. The platform of the media files are using FLV as the main form of communication ${ }^{[5]}$.

\subsection{Technical Detection}

In order to verify the streaming media technology to the "agricultural information" platform advantage provided by ASP.NET Paraller on concurrent stress testing, through the page response time, to determine the degree of user experience. Parallel serial code can be read, by use of this feature to record the first response of the start time and a start time, both of this is the response time 
of the page, and can perform multiple cycles, concurrent.

Using Parallel For () method to achieve multi concurrent. N BackgroundWorker object was created in advance, and the Http request object was pre packaged into the List collection. After the cycle unified activation call, to achieve pressure test.

According to the principle of 8 seconds ( 8 seconds is a famous principle of Internet users access the Web page, if more than 8 seconds will feel impatient, if need to download too long time, they will give up, when concurrent access) 2000 request, the maximum response time of 8 seconds, the average response time for 4.225 seconds; When using the general media to play, the same 2000 requests, the maximum response time of 16.75 seconds, the average response time of 4.322 seconds. Thus, it can be learned that the use of streaming media technology, greatly increasing the amount of concurrent platform, improve the user experience.

\section{System Database Design}

Database is the latest technology of data management, and it is the soul and foundation of software development. Therefore, the success of the database design plays an important role in the design and implementation of the whole system.

\subsection{Database E-R Diagram Design}

Agricultural information service platform consists of six entities, including ordinary users, platform administrators, visitors, science and technology training, policy announcements and message. Visitors through the registration as an ordinary user, you can leave comments and watch training materials. Administrators maintain user information, policy information and training materials. The database E-R diagram of the system is shown in figure 2.

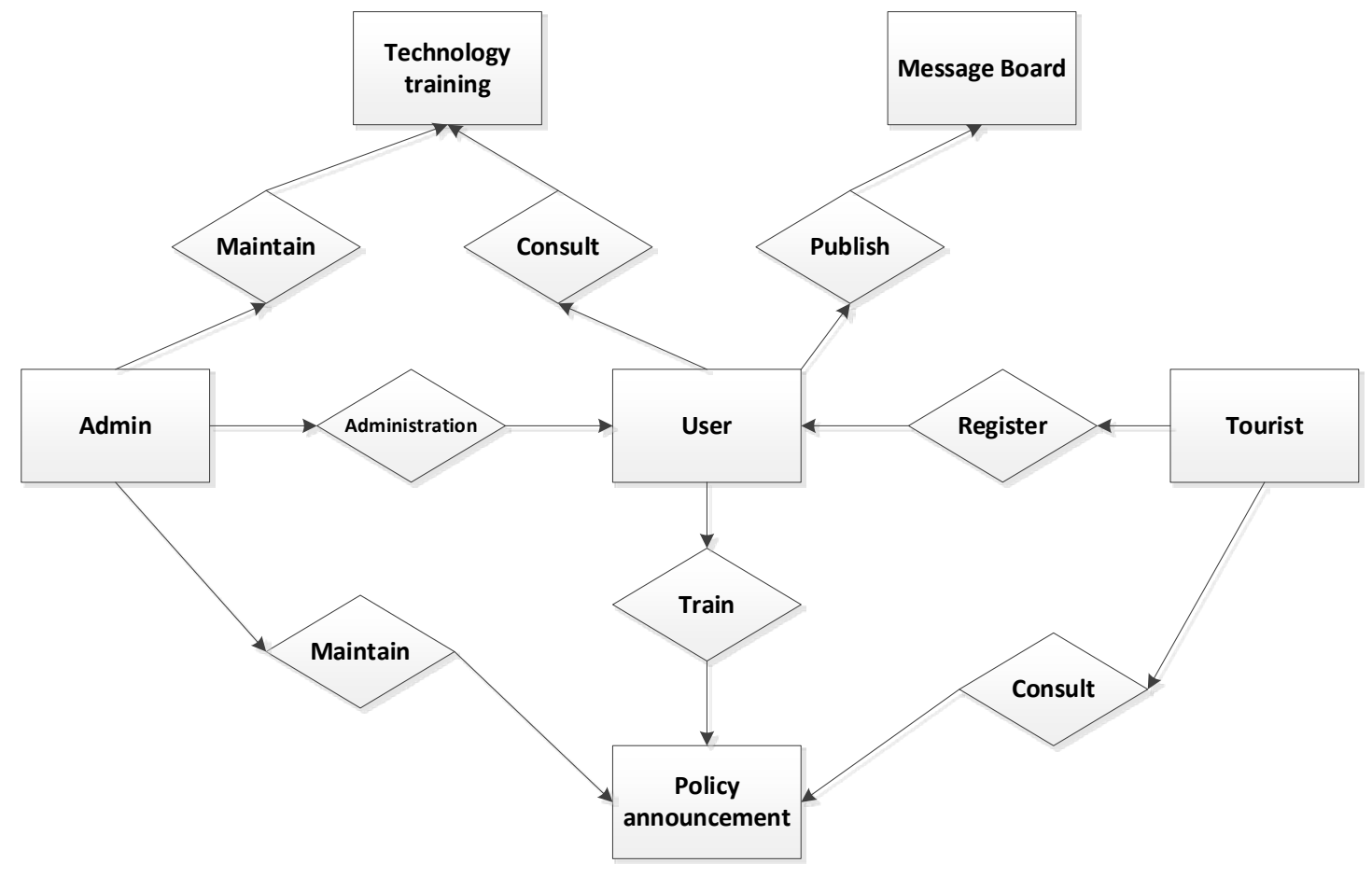

Figure 2. E-R agricultural information service platform 


\subsection{Database Logical Structure Design}

Through the transformation of the E-R diagram to the relational model, the relational model of the agricultural information service platform is obtained, and the optimization of the logical structure of the database is designed according to the characteristics of DBMS:

Policy announcement (policy information number, policy category, upload time, upload, read)

User table (user number, permission type, account name, contact information, gender, true name, date of birth)

Training materials (data number, data name, category number, category name, upload time, upload, reading times)

Policy announcement category (category number, category name)

Message board (message number, user number, message content)

\subsection{Database Physical Structure Design}

Table 2 Policy announcements

\begin{tabular}{cccc}
\hline Name & Type & Length & Explain \\
\hline PolicyID & Int & & Policy number \\
PolicyType & Nvarchar & 10 & Policy category \\
Updatetime & Datetime & & Upload time \\
LoadName & Nvarchar & 10 & Upload \\
Times & Int & & Reading times \\
\hline
\end{tabular}

Table 3 User information

\begin{tabular}{cccc}
\hline Name & Type & Length & Explain \\
\hline UserID & Int & & User number \\
Type & Bit & & Permission type \\
UserName & Nvarchar & 20 & title of account \\
Tel & Nvarchar & 11 & Contact information \\
Sex & Bit & & Gender \\
RealName & Nvarchar & 10 & Real name \\
Birthday & Datetime & & Date of birth \\
\hline
\end{tabular}

Table 4 Training materials

\begin{tabular}{cccc}
\hline Name & Type & Length & Explain \\
\hline TrainID & Int & 50 & Data number \\
TrainName & Nvarchar & & Data name \\
TypeNO & Int & 50 & Class number \\
TypeName & Nvarchar & & Class name \\
UpdateTime & Datetime & \multirow{2}{*}{10} & Upload time \\
LoadName & Nvarchar & & Upload \\
Times & Int & & Reading times \\
\hline
\end{tabular}


Table 5 Categories of policy announcements

\begin{tabular}{cccc}
\hline Name & Type & Length & Explain \\
\hline $\begin{array}{c}\text { TrainTypeID } \\
\text { TypeName }\end{array}$ & Int & 50 & Class number \\
Nvarchar & Class name \\
\hline \multicolumn{2}{c}{ Table 6 Message } \\
Name & Type & Length & Explain \\
\hline MessageID & Int & Message / number \\
UserID & Int & User number \\
Content & Nvarchar & 200 & Message
\end{tabular}

\section{Summary}

In order to accelerate the pace of construction of agricultural informatization, vigorously promote agricultural planting / Breeding and pest control technology, the development of streaming media technology in agricultural information service platform based on fluency operation and low resource share of streaming media and the compatibility of this platform for the actual demand is the key technology, policy announcement module, the platform of science and technology training module and message module, the three for the agricultural sector users, is the three functions of very strong practicability, combined with the "Internet plus" idea of agricultural knowledge dissemination and science, will be able to effectively disseminate the latest consultation to every corner, shorten the actual distance in physical space, and the timely exchange of technology, will promote the learning efficiency of agricultural laborers with.

\section{Reference}

[1] Cai Zhaoquan. Design and implementation of news release system based on .NET[J] Computer Engineering, 2005,26 (6): 1645-1650

[2] Marshall. Visual Studio 2010 parallel programming from entry to master [M]. Beijing: Tsinghua University press, 2013

[3] Yang Hongjun, Yu Tong. Design and implementation of online shopping system based on [J]. VB.NET computer technology, 2011,7 (21): 5077-5079

[4] Tong Xiaonian, Ji Jianan. ASP.net based online examination system design [J]. computer and network, 2005,5:52-53

[5] Wang Kuipeng, Liu Jianhui. FLV file format and its embedded application [J], computer system application, 2010 